\title{
Procedimiento especial de terminación del contrato laboral por bajo desempeño
}

\author{
Recibido: 15 de mayo de 2020 • Aprobado: 11 de junio de 2020 \\ https://doi.org/10.22395/ojum.v20n4la5
}

\author{
Juan Gabriel Tejada Correa \\ Grupo EMI, Medellín, Colombia \\ jgtejada@jgtejadaabogado.com \\ https://orcid.org/0000-0001-5777-4718
}

\section{RESUMEN}

El presente artículo se propone revisar las condiciones para despedir un trabajador, específicamente por su bajo desempeño, en aras de determinar un procedimiento claro, seguro y eficaz que permita mejorar el rendimiento de dichos trabajadores en las organizaciones de naturaleza privada y respetar sus derechos fundamentales. La metodología de esta investigación es hermenéutico-documental. Se desarrolla a través de rastreos bibliográficos, recopilación de literatura sobre administración de personal y análisis e interpretación de la legislación y jurisprudencia regulatoria de la materia. Como conclusión principal, se obtiene que será válido, seguro y eficaz terminar el contrato de trabajo con justa causa si se da aplicación a un procedimiento que tenga como insumo principal la evaluación del desempeño ejecutada en la empresa, seguido por un proceso de retroalimentación que brinde herramientas para el mejoramiento del desempeño e indague por aspectos propios de la esfera personal del trabajador que potencialmente afecten la prestación del servicio, y finalice con un proceso disciplinario respetuoso del derecho fundamental al debido proceso.

Palabras clave: derechos fundamentales del trabajador; desempeño laboral; productividad laboral; terminación del contrato. 


\section{Special Procedure for Termination of Employment Contract Due to Poor Performance}

\section{ABSTRACT}

The purpose of this article is to review the conditions for dismissing an employee, specifically for poor performance, in order to determine a clear, safe and effective procedure to improve the performance of such employees in private organizations and to respect their fundamental rights. The methodology of this research is hermeneutic-documentary. It is developed through bibliographic searches, compilation of literature on personnel administration and analysis and interpretation of legislation and regulatory jurisprudence on the subject. As main conclusion, it is obtained that it will be valid, safe and effective to terminate the employment contract with just cause if a procedure is applied that has as main input the performance evaluation carried out in the company, followed by a feedback process that provides tools for performance improvement and inquires about aspects of the employee's personal sphere that potentially affect the provision of the service, and ends with a disciplinary process respectful of the fundamental right to due process.

Keywords: fundamental rights of the worker; labor performance; labor productivity; termination of contract.

\section{Procedimento especial para rescisão do contrato de trabalho por mau desempenho}

\section{RESUMO}

Este artigo visa rever as condições de demissão de um trabalhador, especificamente por mau desempenho, a fim de determinar um procedimento claro, seguro e eficaz para melhorar o desempenho de tais trabalhadores em organizações privadas e respeitar seus direitos fundamentais. A metodologia desta pesquisa é hermenêuticodocumentário. Ela é desenvolvida através de rastreamento bibliográfico, compilação de literatura sobre administração de pessoal e análise e interpretação da legislação e jurisprudência reguladora do assunto. Como conclusão principal, obtém-se que será válido, seguro e eficaz rescindir o contrato de trabalho com justa causa se for aplicado um procedimento que tenha como principal insumo a avaliação de desempenho realizada na empresa, seguido de um processo de feedback que forneça ferramentas para a melhoria do desempenho e indague sobre aspectos da esfera pessoal do trabalhador que potencialmente afetam a prestação do serviço, e termina com um processo disciplinar que respeite o direito fundamental ao devido processo.

Palavras-chave: direitos fundamentais do trabalhador; desempenho do trabalho; produtividade do trabalho; rescisão do contrato. 


\section{INTRODUCCIÓN}

La Corte Constitucional (Sentencia C-593, 2014) ha sido clara en indicar los requisitos mínimos que el empleador debe cumplir para sancionar válidamente a un trabajador garantizando el debido proceso. Sin embargo, no es suficientemente claro el procedimiento que se debe aplicar para efectos de despedir un trabajador por bajo desempeño laboral, que es el tema por desarrollar. En consecuencia, para profundizar esta materia, se propone a lo largo de este trabajo, producto de una investigación para optar al título de magíster en Derecho, revisar las condiciones para despedir un trabajador específicamente por su bajo desempeño en aras de determinar un procedimiento claro, seguro y eficaz que permita mejorar el rendimiento de dichos trabajadores en las organizaciones de naturaleza privada y respetar sus derechos fundamentales, lo que se traduciría en el mejoramiento continuo de la productividad de la empresa.

La relevancia de la presente investigación radica en que en ella se proponen mecanismos jurídicamente válidos y respetuosos de la dignidad de los trabajadores, orientados a mejorar el rendimiento de su desempeño laboral. Este objetivo responde a que es necesario conjugar el impulso constante de las empresas por mejorar la eficiencia de sus procesos, con las normas legales y reglas jurisprudenciales que limitan las facultades del empleador para despedir un trabajador.

Así, la utilidad de este trabajo se fundamenta en que sus conclusiones están orientadas a la construcción de un procedimiento enfocado en despedir válidamente a un trabajador con ocasión de su bajo desempeño laboral para buscar mejorar constantemente la productividad de las empresas a través de decisiones respetuosas del ser humano, objetivas, seguras que minimicen los riesgos legales propios de las relaciones de trabajo. En esta contribución se desarrolla un procedimiento fundamentado, en primer lugar, en las teorías sobre evaluación del desempeño, en las que son formados los profesionales en psicología, administración e ingeniería, que lideran y conforman las áreas de recursos humanos en las empresas. En segundo lugar, se basa en la legislación y jurisprudencia colombiana que determinan los procesos a ejecutar para despedir válidamente a un trabajador en caso de que registre bajo desempeño en las funciones que le fueron asignadas.

La metodología de esta investigación es hermenéutico-documental, dado que se desarrolló a través de rastreos bibliográficos, recopilación de literatura sobre administración de personal y análisis e interpretación de legislación y jurisprudencia regulatoria de la materia, de acuerdo con las necesidades específicas del procedimiento laboral que aquí se aborda.

Finalmente, este estudio investigativo se compone de tres partes. En la primera se identifican los fundamentos del proceso de evaluación del desempeño adoptado actualmente por las empresas, en la segunda se determinan las condiciones legales 
y jurisprudenciales que limitan las facultades del empleador para despedir a un trabajador por las razones de bajo desempeño y, en la tercera, se indican las etapas a ejecutar para despedir un trabajador en virtud de su bajo desempeño laboral. Y la tercera es, puntualmente, la propuesta de investigación con la que se espera hacer un aporte al campo del derecho laboral.

\section{FUNDAMENTOS DEL PROCESO DE EVALUACIÓN DEL DESEMPEÑO}

Previo al análisis de las normas legales y las reglas jurisprudenciales que sistematizan las medidas que el empleador puede aplicar para terminar el contrato de trabajo en virtud del bajo rendimiento de un empleado, es necesario analizar el concepto de desempeño laboral y su conexión con la productividad de una empresa.

El desempeño de los trabajadores cobra cada día mayor importancia, y las organizaciones han tomado consciencia de que este es un elemento fundamental que el proceso de dirección estratégica de una empresa debe considerar cuidadosamente. En este sentido, es necesario conocer cómo están desempeñando sus labores los empleados, a fin de identificar quiénes efectivamente agregan valor a los procesos productivos y quiénes no (Sanchez y Calderon, 2012).

En aras de este objetivo, las empresas llevan a cabo las evaluaciones de desempeño de sus trabajadores con el propósito de asignar calificaciones a los empleados. De esta manera, pueden discriminar entre empleados efectivos y empleados no efectivos (Sanchez y Calderon, 2012).

Al decir lo anterior, se pone en evidencia la relación entre desempeño y productividad que surge de la interrelación existente entre personas y organizaciones, dado que las personas se emplean en las organizaciones para satisfacer sus necesidades y las organizaciones necesitan de las personas para funcionar y obtener más y mejores resultados (Cequea, Rodriguez y Nuñez, 2011). Esta misma relación entre desempeño y productividad aparece desde la definición que hace Adam Smith (1996) del concepto de trabajo. En Una mirada a la productividad laboral para las pymes de confecciones, Jaimes y Rojas (2015) permiten comprender que el filósofo y economista escocés concibe el elemento del trabajo como un factor de producción junto con la tierra, el cual es realizado por personas. Esto quiere decir que, ya desde Smith (1996), se valora el impacto de los operarios en la productividad que ofrezca la tierra o la empresa, principalmente debido a la constante mejora que las personas hacen de sus habilidades para el trabajo.

Ahora bien, si se entiende productividad como la forma de utilizar los factores de producción en la generación de bienes y servicios para la sociedad y, simultáneamente, como la búsqueda de mejorar la eficiencia y la eficacia con que son utilizados los recursos (Medina, 2010) - y teniendo en cuenta la relación antes mencionada entre desempeño laboral y productividad-, es coherente, entonces, que aparezca la evaluación del desempeño laboral en las actuales relaciones laborales. 
En este orden de ideas, la evaluación de desempeño refleja principalmente la medida en que se cumplen los objetivos de eficiencia, esto es, de alcanzar las metas al tiempo que se utilizan los recursos eficientemente con el fin de descubrir en qué medida es productivo el empleado y si podrá mejorar su rendimiento futuro (Chiang y San Martín, 2017).

En consecuencia, el rendimiento laboral está necesariamente relacionado con la eficiencia, lo que lo convierte en una variable a estudiar dado que impacta el éxito en la obtención de resultados y el cumplimiento de objetivos propuestos por la organización. Se entiende, entonces, la evaluación del rendimiento laboral como un eje central en la gestión empresarial que se relaciona directamente con la productividad de la empresa, de ahí la importancia de prever e incluso controlar variables que interfieren en el rendimiento laboral de los empleados (Córdoba y Moreno, 2013).

Lo dicho hasta el momento permite comprender que la evaluación de desempeño es un proceso clave que consiste en valorar de forma sistemática y objetiva el rendimiento de los empleados. Esta tiene como objetivo principal la mejora de la actuación de cada uno de ellos en el campo de trabajo para lograr un aumento en la productividad empresarial y el incremento del buen rendimiento laboral de cada empleado (Cuesta, 2017). En este sentido, la evaluación de desempeño se define como la medición sistemática del grado de eficacia y eficiencia con el que los trabajadores realizan sus actividades laborales durante un periodo determinado y su potencial desarrollo, pues así se construye una base para elaborar y ejecutar planes individuales de capacitación y desarrollo de los diferentes miembros que conforman la organización (Cuesta et al., 2018). En este sentido, es claro que esta evaluación tiene una visión hacia el pasado y otra visión hacia el futuro, y pretende integrar en mayor grado los objetivos de la empresa con los objetivos de los trabajadores (Rios Giraldo, 2016).

Actualmente, es posible ver que existe consenso entre los autores contemporáneos que han escrito sobre el tema en relación con la visión del concepto técnico de evaluación de desempeño. Este concepto describe una forma de medir el aporte del trabajador al logro de los objetivos empresariales. Los indicadores de las evaluaciones de desempeño deben permitir la medición cuantitativa de algunas actividades de los trabajadores con el fin de analizar qué aspectos deben abordarse para mejorar algún proceso (Arango et al., 2017).

Frente al problema del bajo desempeño laboral que pueda presentar un empleado, los teóricos contemporáneos del tema arguyen que las empresas deben propender por una solución que contenga los siguientes elementos: primero, medición de resultados del trabajo individual y grupal basada en resultados del proceso; segundo, comunicación abierta de los resultados de la medición; tercero, sistema de recompensa, tanto individual como grupal, basado en dichos resultados; cuarto, método de trabajo conjunto entre las personas; y quinto, un método que permita definir y asegurar, 
para cada puesto de trabajo, la persona correcta con la información requerida para desempeñar eficazmente el cargo (Fonseca, 2015).

Ahora bien, sintetizando los fundamentos de los procesos de evaluación de desempeño a partir de los conceptos expuestos anteriormente, puede indicarse que dicha evaluación debe cumplir los siguientes requisitos:

1. Su objetivo debe ser medir el aporte de valor de cada empleado respecto del cumplimiento de los objetivos de la organización.

2. Es una medición objetiva y sistemática para evaluar la eficiencia del empleado en el desempeño de sus funciones.

3. Realiza la medición en periodos de tiempo determinados en aras de observar la evolución de los resultados obtenidos por el trabajador.

4. Tiene por objetivo identificar las oportunidades de mejora de los empleados en su actuación.

5. Aporta sus resultados como insumo objetivo para asignar calificaciones a los empleados y así determinar las acciones a aplicar tanto a los que son efectivos como a los que no lo son.

\section{LIMITACIONES LEGALES Y JURISPRUDENCIALES PARA SANCIONAR EL BAJO DESEMPEÑO LABORAL}

El contrato de trabajo está definido en la legislación colombiana en el artículo 22 del Código Sustantivo de Trabajo como "aquel por el cual una persona natural se obliga a prestar un servicio personal a otra persona, natural o jurídica, bajo la continuada dependencia o subordinación de la segunda y mediante remuneración" (Decreto Ley 2663, 1950). Teniendo en cuenta esta definición, es importante hacer énfasis en el elemento esencial de todo contrato de trabajo, como lo es la subordinación, dado que este elemento es el que otorga las facultades al empleador para sancionar a sus trabajadores cuando sea procedente. Por ende, la subordinación ha sido definida por la Corte Constitucional en los siguientes términos:

La subordinación del trabajador al empleador como elemento distintivo y definidor del contrato de trabajo ha sido entendida, según la concepción más aceptable por la doctrina y la jurisprudencia, como un poder jurídico permanente de que es titular el empleador para dirigir la actividad laboral del trabajador, a través de la expedición de órdenes e instrucciones y la imposición de reglamentos, en lo relativo a la manera como éste debe realizar las funciones y cumplir con las obligaciones que le son propias, con miras al cumplimiento de los objetivos de la empresa, los cuales son generalmente económicos. (Sentencia C-386, 2000)

A partir de estas aclaraciones y definiciones, se ha desarrollado el concepto del poder de dirección del empleador. Este se define como una multiplicidad de facultades 
que otorga el ordenamiento jurídico y que son necesarias e indispensables para el funcionamiento normal de la empresa, su organización económica, técnica y funcional, con el propósito de que el empleador ejerza las facultades de dirigir, dar órdenes e instrucciones, reglamentar la prestación del trabajador en la empresa y de vigilar y sancionar las faltas cometidas por el trabajador (Hernandez, 1997).

En este orden de ideas, la misma Corte Constitucional explica en la Sentencia T-584 (1998) que la expedición de una orden por parte del empleador implica el ejercicio razonable de las respectivas competencias, con la correlativa obligación del trabajador de cumplirla. De manera que, en caso de desobediencia de este, inmediatamente nace para el empleador la posibilidad de aplicar medidas de corrección, entre las cuales están los llamados de atención, las sanciones, suspensiones, la terminación del contrato, entre otros, según la magnitud de la conducta analizada en concreto.

Sin embargo, la jurisprudencia constitucional también ha desarrollado una serie de limitaciones a estas facultades, basadas fundamentalmente en la obligación que tiene el empleador de no considerar al trabajador como un factor de producción, sino como un ser humano merecedor de igual consideración y respeto que cualquier otro ser humano sin importar su estatus social o su oficio (Sentencia T-611, 2001). Adicionalmente, se establece que, como consecuencia de esta subordinación jurídica, el empleador está facultado para exigir el cumplimiento de instrucciones en cualquier momento respecto al modo, tiempo o cantidad de trabajo, y aplicar reglamentos internos de trabajo; solo está limitado por el honor, la dignidad y los derechos mínimos del trabajador (Sentencia C-1110, 2001).

De forma genérica, es claro que existen limitaciones en el ejercicio de las funciones del empleador que se han descrito antes, tal como lo exponen las sentencias de la Corte Constitucional que se han citado. Los derechos fundamentales al debido proceso y al trabajo en condiciones dignas y justas imponen obligaciones específicas al empleador, toda vez que estos derechos exigen de forma expresa condiciones de validez a las acciones del empleador al momento de ejercer la facultad disciplinaria y, más aún, cuando se busca terminar el contrato de trabajo con ocasión del bajo desempeño laboral. En tal sentido, en aras de hacer efectivas las garantías consagradas en la Constitución Política, la Corte Constitucional estableció en la Sentencia C-593 (2014) que es indispensable que los entes de carácter privado fijen unas formas o parámetros mínimos que delimiten el uso de la facultad disciplinaria que, además, ha agregado que tales procedimientos deben asegurar al menos:

a) La comunicación formal de la apertura del proceso disciplinario a la persona a quien se imputan las conductas posibles de sanción; b) la formulación de los cargos imputados, que puede ser verbal o escrita, siempre y cuando en ella consten de manera clara y precisa las conductas, las faltas disciplinarias a que esas conductas dan lugar y la calificación provisional de las conductas como faltas disciplinarias; c) el traslado al imputado de todas y cada una de las pruebas que 
fundamentan los cargos formulados; d) la indicación de un término durante el cual el acusado pueda formular sus descargos, controvertir las pruebas en su contra y allegar las que considere necesarias para sustentar sus descargos; e) el pronunciamiento definitivo de las autoridades competentes mediante un acto motivado y congruente; f) la imposición de una sanción proporcional a los hechos que la motivaron; y g) la posibilidad de que el encartado pueda controvertir, mediante los recursos pertinentes, todas y cada una de las decisiones. (Sentencia C-593, 2014)

De esta forma, se establecieron claramente los parámetros mínimos para expedir el respectivo procedimiento de ejecución obligatoria por parte del empleador para sancionar cualquier falta disciplinaria en la que incurra un trabajador. En este sentido, es importante destacar que el desconocimiento de estos, dejará sin efecto la sanción que se imponga a un trabajador, quien podrá instaurar una acción de tutela solicitando el amparo de su derecho al debido proceso. Por tanto, la única protección efectiva para el empleador es respetar este derecho fundamental dentro del procedimiento que tenga establecido, puesto que el fin último de este principio es prevenir la arbitrariedad, término definido como el acto o proceder contrario a la justicia, la razón o las leyes, dictado solo por la voluntad o el capricho (Tejada, 2016).

Adicionalmente, dado que el desempeño laboral es la consecuencia directa de las conductas de los trabajadores, se debe cumplir la obligación que tiene el empleador de no considerar al trabajador como un factor de producción, sino como un ser humano merecedor de consideración y respeto en aplicación del derecho al trabajo en condiciones dignas y justas precisamente cuando se aplica una sanción, tal como lo menciona la Corte Constitucional (Sentencia T-611, 2001). En este sentido, el empleador debe tener presente que la potestad disciplinaria no lo reviste de atribuciones omnímodas que le permitan tomar al trabajador como una simple pieza que integra la totalidad que representa la empresa, sino que debe entenderlo como un ser humano libre, responsable y digno, en quien debe cristalizarse la administración de justicia distributiva a cargo del patrono (Sentencia T-682, 2014).

La precisión que aparece en el párrafo anterior obliga a que el empleador indague sobre las condiciones, situaciones o circunstancias personales - tales como la situación familiar, el estado de salud del trabajador o su núcleo familiar (Sentencia T-509, 2019) — que pueden afectar las capacidades y la concentración del trabajador y obstruir su óptimo desempeño. Esta claridad configura, sin duda alguna, una limitante más al ejercicio de las facultades del empleador, quien en cada situación que se presente con un trabajador deberá apreciar el conjunto de estos elementos y adoptar una determinación que los consulte de manera adecuada y coherente para evitar violentar los derechos fundamentales de sus empleados (Sentencia T-483, 1993).

Por su parte, la Corte Suprema de Justicia también ha establecido en su jurisprudencia las limitaciones que tiene el empleador en el ejercicio de las facultades que le otorga la ley. En este contexto, señala que en la Constitución Política se reconoce 
el derecho a la libertad de empresa, la cual consiste en la posibilidad que tiene todo individuo de ejecutar una actividad económica y de estructurar a su manera dicha organización, lo que incluye la dirección de las relaciones con sus trabajadores y si para ello contrata personas para que le presten sus servicios. Sin embargo, esas facultades empresariales no son absolutas y están limitadas por los derechos constitucionales, las condiciones dignas y justas que deben orientar todo contrato de trabajo y por los principios de buena fe, solidaridad, dignidad, igualdad y función social de la empresa (Sentencia SL3424, 2018).

La primera limitación es la temporalidad para notificar la terminación del contrato. Frente a ello, la Corte Suprema de Justicia ha señalado que la terminación unilateral y por justa causa del contrato de trabajo por parte del empleador debe ser oportuna. Si bien en la legislación no ha establecido límites temporales máximos para notificar la decisión de dar por terminado el contrato una vez se identifique una conducta del trabajador que configure una falta que dé lugar a su adopción, se exige que dicha decisión se adopte en un término razonable entre lo uno y lo otro (Sentencia SL1146, 2018).

En ese contexto, el despido se entiende como la decisión unilateral del empleador de dar por terminado un contrato de trabajo cuando una conducta del trabajador configura el supuesto de hecho de las causales determinadas en la ley laboral, cuyo propósito es asegurar su legalidad. Estas causales van acompañadas, en algunos casos, de formalidades que regulan el procedimiento a seguir, es decir, regulan la decisión a aplicar y protegen al trabajador contra posibles medidas arbitrarias por parte del empleador, lo que configuran el denominado debido proceso (Sentencia SL3424, 2018).

En este sentido y de forma específica cuando se trata de dar aplicación a la causal de terminación del contrato de trabajo establecida en el numeral 9 del artículo 62 del Código Sustantivo del Trabajo (Decreto Ley 2663, 1950), la Corte Suprema de Justicia ha establecido que para dar aplicación a esta causal es necesario agotar el procedimiento establecido en el Decreto 1373 (1966) para que esta causal sea legalmente procedente. Esta formalidad tiene el objetivo de brindar la oportunidad al trabajador de que corrija su rendimiento antes de dar por terminado el contrato (Sentencia 35516, 2009). Asimismo, la jurisprudencia de esta alta corte estipula que la omisión de este procedimiento conlleva a que la terminación del contrato de trabajo sea injusta y, por ende, se debe pagar la indemnización establecida en el artículo 64 del Código Sustantivo de Trabajo (Sentencia SL023, 2018).

En este orden de ideas, es necesario establecer si el despido que se pretende ejecutar no es arbitrario y cumple con la totalidad de requisitos legales, dejando claro que siempre se debe verificar de manera previa si el trabajador es: a) dirigente sindical o amparado por fuero circunstancial, b) persona con discapacidad, c) madre gestante, d) amparado por fuero de paternidad, e) prepensionado, f) padre o madre cabeza de 
familia, g) secuestrado, h) denunciante de acoso laboral, o i) si con el despido se están violando derechos fundamentales del trabajador. Las anteriores condiciones generan una protección reforzada que impide la terminación del contrato de trabajo mediante acto unilateral del empleador. Por ende, en caso de despedir un trabajador inmerso en alguna de estas condiciones, este deviene en ineficaz y no produce efectos legales, lo que lleva a que eventualmente se ordene su reintegro mediante sentencia judicial (Sentencia 174, 2019)

Aunado a las anteriores limitaciones que aplican en términos generales a cualquier despido que se ejecute, existen adicionalmente condicionamientos procedimentales específicos que establece la legislación laboral para que proceda un despido por bajo desempeño, conforme a lo estipulado en el numeral 9 del artículo 62 del Código Sustantivo de Trabajo (Decreto Ley 2663, 1950): "El deficiente rendimiento en el trabajo en relación con la capacidad del trabajador y con el rendimiento promedio en labores análogas, cuando no se corrija en un plazo razonable a pesar del requerimiento del empleador".

De la racionalidad de esta norma se observa que es necesario conceder un plazo razonable al trabajador para que ejecute acciones que tiendan al mejoramiento de su rendimiento laboral. Por ende, esta causal de terminación del contrato de trabajo fue reglamentada por el Decreto 1373 (1966) que agregó las siguientes condiciones para terminar un contrato de trabajo por bajo rendimiento laboral:

Para dar aplicación al numeral 9) del artículo $7^{\circ}$ del Decreto 2351 de 1965, el patrono deberá ceñirse al siguiente procedimiento:

a) Requerirá al trabajador dos (2) veces, cuando menos, por escrito, mediando entre uno y otro requerimiento un lapso no inferior a ocho (8) días;

b) Si hechos los anteriores requerimientos el patrono considera que aún subsiste el deficiente rendimiento laboral del trabajador, presentará a éste un cuadro comparativo de rendimiento promedio en actividades análogas, a efecto de que el trabajador pueda presentar sus descargos por escrito dentro de los ocho (8) días siguientes; y

c) Si el patrono no quedare conforme con las justificaciones del trabajador, así se lo hará saber por escrito dentro de los ocho (8) días siguientes. (art. 9)

Ahora bien, esta norma configura una limitación a las facultades del empleador para terminar el contrato de trabajo, frente a lo cual la Corte Suprema de Justicia ha señalado que es necesario agotar este trámite que ha de adelantarse en caso de rendimiento deficiente del trabajador y así aplicar la causal de terminación del contrato de trabajo establecida en el numeral 9 del artículo 62 del Código Sustantivo de Trabajo (Sentencia 40283, 2011). 
Adicionalmente, el mismo artículo 62 en su numeral 9 impone un requisito más para aplicar esta causal de terminación del contrato, al establecer que "en los casos de los numerales 9 a 15 de este artículo, para la terminación del contrato, el lempleadorl deberá dar aviso al trabajador con anticipación no menor de quince (15) días" (Decreto Ley 2663, 1950). Esto implica una de dos opciones: la primera es notificar al trabajador la terminación de su contrato por bajo rendimiento y programar la fecha de finalización del contrato quince días después. La segunda es pagar, adicional a la liquidación final de prestaciones sociales, quince días de salario al trabajador. Ambas opciones son claras afectaciones a la eficiencia operativa de la compañía.

Lo anterior se destaca dado que la Corte Suprema de Justicia ha establecido tradicionalmente que no es necesario realizar un proceso disciplinario ni citar el trabajador a descargos para efectos de notificar la terminación del contrato con justa causa. En este sentido, señala que como la citación a descargos no está prevista en la ley como requisito previo al despido, lo mínimo legalmente exigible es que, al momento del retiro, se le haga saber a este los motivos y razones concretas del despido que se suponen han sido previamente establecidas por el empleador, con o sin descargos del trabajador, y que, en todo caso, este haya tenido la oportunidad de conocer y controvertir los hechos que pueden ser constitutivos de la resolución del contrato con justa causa; la oportunidad de contradicción no se surte necesaria o únicamente con una citación a descargos (Sentencia SL1444, 2018). Pero como se puede observar en el caso del bajo rendimiento, existe norma expresa que efectivamente exige la citación a descargos como requisito para dar aplicación a esta causal de terminación del contrato.

Lo dicho hasta el momento deja claro que frente al bajo desempeño laboral se tienen limitaciones adicionales a las de cualquier otra causal de terminación del contrato de trabajo, lo cual genera la necesidad de estudiar con mayor profundidad estas condiciones procedimentales en aras de garantizar la eficacia y validez de las decisiones a adoptar con el propósito de mejorar la productividad de las empresas. La siguiente sección se dirige, precisamente, a dicha profundización.

En atención a todo lo anterior, y haciendo énfasis en materia de desempeño laboral y aplicando los fundamentos expuestos en la primera parte, se puede considerar que el bajo rendimiento laboral de un empleado configura un incumplimiento laboral cuando el trabajador registra bajos resultados en las mediciones aplicadas de forma periódica y sistemática, y cuando se demuestra que no hay una evolución positiva en su desempeño en determinados periodos de evaluación.

De este modo, para describir las condiciones de tiempo, modo y lugar del bajo rendimiento laboral se requiere como mínimo a) periodos determinados para la aplicación de las evaluaciones, b) resultados de las evaluaciones aplicadas y c) reiteración de bajos resultados en las evaluaciones. Con estos elementos, es posible afirmar que una decisión de carácter sancionatorio garantiza el derecho fundamental al debido 
proceso, sin perjuicio del deber de desarrollar un proceso disciplinario que cumpla con los parámetros mínimos establecidos en la Sentencia C-593 (2014) ya mencionados.

\section{PROCEDIMIENTO PARA DESPEDIR VÁLLDAMENTE A UN EMPLEADO EN VIRTUD DEL BAJO DESEMPEÑO LABORAL}

Previo a la descripción del procedimiento que debe ejecutarse para despedir válida, segura y eficazmente a un empleado en virtud de su bajo desempeño laboral, es fundamental considerar que este procedimiento hace parte de la disciplina laboral de la empresa, entendida esta como el cabal cumplimiento de las funciones del cargo o puesto de trabajo en concordancia con las metas corporativas sobre la base de normas y procedimientos dados por la cultura organizacional (Cuesta, 2017). Por su parte, Castillo (1993) ha definido como disciplina laboral el conjunto de acciones orientadas a lograr el acatamiento de las políticas, normas y reglamentos de la empresa por parte de todos los miembros de la organización.

Desde la perspectiva de Chiavenato (2008), el término disciplina laboral significaba simplemente que las personas se alinearan a las reglas y normas que la organización establecía, porque eran adecuadas para alcanzar los objetivos de la organización. Ahora bien, debido a este concepto de disciplina, las organizaciones enfocaban su gestión en controlar los medios y olvidaban los fines. Por ende, al vigilar conductas relacionadas con la puntualidad, la frecuencia y la reiteración, la obediencia al jefe y el orden, las empresas dejaban de enfocarse en aspectos importantes tales como la eficiencia, la eficacia de cada trabajador, la relación del cumplimiento de funciones frente al logro de las metas y objetivos, el valor agregado a la organización, la mejora de la calidad y de la productividad, la atención al cliente y otros aspectos de valor real para la organización (Chiavenato, 2008).

En la actualidad debe entenderse la disciplina laboral como aquella acción organizacional que se ejecuta para alentar y garantizar el cumplimiento de las normas internas vigentes en una empresa. Es un tipo de capacitación orientada a corregir y moldear las actividades y la conducta de los empleados para que los esfuerzos individuales se encaucen mejor, en aras de la cooperación y el desempeño. Quedan claras, entonces, las acciones disciplinarias negativas o correctivas que deben considerarse como una oportunidad de aprendizaje y mejoramiento para las partes involucradas, dado que el propósito de estas acciones es garantizar que el comportamiento y el desempeño de los trabajadores estén alineados con los objetivos y normatividad de la compañía (Bohlander, Snell y Herrero, 2008).

Para el estudio que propongo en este apartado es necesario analizar el concepto de productividad, dado que el objetivo trazado es desarrollar estrategias de disciplina laboral para el mejoramiento del desempeño y, por ende, el mejoramiento de la productividad empresarial. En consecuencia, se entiende en esta investigación por productividad 
la medida de eficiencia con la que se utiliza el trabajo y el capital para producir valor económico (Galindo y Rios, 2015), tal como aparece ya definida en el primer apartado de este trabajo. Se entiende también que se habla de productividad como la relación existente entre la cantidad de bienes y servicios producidos y la cantidad de recursos utilizados. De esta manera, la productividad en términos de trabajo es sinónimo de rendimiento y eficiencia (Buitrago, 2013). Ahora bien, en términos más simples, la productividad se entiende como la capacidad de hacer más con menos, obtener más resultados con los mismos insumos (Fonseca, 2015).

De acuerdo con lo dicho, y descendiendo a los aspectos jurídicos del tema de investigación, se reitera que las normas que regulan las facultades para despedir un trabajador en virtud de su bajo desempeño son el Código Sustantivo del Trabajo (Decreto Ley 2663, 1950art. 62, num. 9), y su Decreto 1373 (1966, art. 2); Estas normas obligan a cumplir un procedimiento específico que se analiza y explica a continuación:

\section{Requerimientos previos al trabajador}

La primera obligación del empleador en estos casos implica requerir previamente al trabajador como mínimo en dos ocasiones (Decreto 1373, 1966 art. 2, lit. a) para notificarle los resultados obtenidos en las evaluaciones de desempeño aplicadas con anterioridad. En este punto, la norma claramente obliga al empleador a aplicar evaluaciones de desempeño previas y periódicas para obtener el soporte probatorio necesario que le permita sustentar ante el colaborador, y posteriormente ante jueces y autoridades administrativas, que sus resultados no son óptimos ni eficientes en el desarrollo de sus funciones. Esta evaluación debe dar cumplimiento a los fundamentos expuestos en el primera sección.

La finalidad de este requerimiento es retroalimentar a los trabajadores respecto de los resultados obtenidos en las evaluaciones de desempeño que le han sido aplicadas en períodos específicos, cuyos resultados son claramente más bajos comparados con los de quienes desarrollan actividades análogas, es decir, se trata de comparar los resultados obtenidos por cada trabajador frente a los resultados de sus pares o frente a los resultados de aquellos empleados que desarrollen funciones semejantes. El requerimiento del que se habla se hace en virtud de la necesidad de informar, en primer lugar, los resultados obtenidos por cada persona durante las evaluaciones de desempeño que se realizan y, en segundo lugar, brindar las recomendaciones correspondientes para que el trabajador desarrolle sus funciones de forma más eficiente. Además de esto, esta retroalimentación se debe entender como una conversación para la coordinación de acciones futuras que permitan modificar los resultados obtenidos por el trabajador. Su objetivo es lograr el mejoramiento de su desempeño, escuchar el punto de vista del trabajador, examinar las razones por las que está obteniendo 
dichos resultados insatisfactorios e indagar por qué realiza determinadas acciones que no aportan a una labor bien realizada (Echeverria, 2003).

Para este tipo de conversaciones, autores como Yepes (2017) y Bock (2015) señalan que toda conversación admite debate, dialéctica, contraposición, discusión y, sobre todo, consenso. Por esta razón, no se trata de una advertencia marcada por la autoridad: "o te pones las pilas, o te largas", sino que se trata de un diálogo formativo y edificante para ayudar a progresar. Realizadas de este modo, y a nivel individual, la retroalimentación del desempeño es bien aceptada; se la comprende como la intervención más efectiva disponible para mejorar el aprendizaje y el rendimiento laboral (Eisele, 2012).

En esta etapa del proceso de requerimiento, descrita como un diálogo formativo y edificante, es de vital importancia indagar por situaciones de índole personal, familiar o conyugal, duelos, condiciones de salud, problemas financieros, legales, adicciones, depresión, ansiedad, entre otras (Chiavenato, 2008). El objetivo de ello es determinar si estas situaciones están afectando al trabajador a tal punto que son la causa de su bajo desempeño, tal como lo exige la jurisprudencia de la Corte Constitucional (Sentencia T-483, 1993). Dicha sentencia señala que la discrecionalidad del empleador está limitada por el derecho al trabajo en condiciones dignas y justas. De esta manera, reitera que este derecho exige la evaluación de circunstancias de índole personal que afectan al trabajador para efectos de adoptar la decisión más justa respecto de este colaborador, ya que de detectarse este tipo de novedades de índole personal, es necesario brindarle asistencia al trabajador y adaptar las condiciones de trabajo y de evaluación de acuerdo con sus condiciones específicas; todo esto debe ser debidamente informado al trabajador en cuestión.

Indagar por los aspectos personales o aspectos humanos es importante, toda vez que el hecho productivo requiere de la participación de las personas, de una permanente relación social laboral, lo que implica aspectos tanto objetivos como subjetivos y, en consecuencia, "la productividad de una organización está afectada por ciertas características y comportamientos del individuo en las cuales están inmersos procesos psicológicos y psicosociales" (Cequea, Rodriguez y Nuñez, 2011, p. 555).

Ahora bien, estas retroalimentaciones y diálogos se deben realizar sobre un grupo delimitado de colaboradores que registren los peores resultados en su evaluación de desempeño. Se recomiendan grupos de entre el 5 \% y el 20 \% del grupo de trabajadores que estén bajo la misma línea de mando de un Jefe Inmediato (Bock, 2015) y focalizar esta acción en quienes efectivamente registran el rendimiento más deficiente. Es necesario indicar que la realización de una retroalimentación requiere preparación, dado que es necesario considerar y preparar cómo plantear la situación para evitar frases desmedidas que puedan generar reacciones negativas (Bermudez et al., 2016), ya que las mismas palabras se pueden entender como un consejo, una 
orden o una amenaza (Gomez, 1991). Finalmente, es prioritario dejar registro de la realización de este procedimiento como constancia para su seguimiento.

Se destaca que esta norma establece que deben realizarse, como mínimo, dos de estos requerimientos en un periodo de ocho días antes de iniciar el proceso disciplinario a través del cual se procederá a despedir al trabajador. En consecuencia, de la racionalidad de la norma se observa que claramente el propósito aquí es informar al trabajador los resultados negativos que arrojan las mediciones de su desempeño, pues el propósito de las evaluaciones de desempeño es identificar oportunidades de mejora en la actuación del trabajador e informarlas debida y oportunamente. Consecuentemente, esta evaluación es sistemática en periodos definidos y conocidos previamente por el trabajador para que desarrolle acciones orientadas a incrementar su rendimiento.

\section{Análisis comparativo de rendimiento}

El siguiente requisito exigido en el literal b del artículo 2 del Decreto 1373 (1966), se relaciona con la obligación de presentar un cuadro comparativo de desempeño del colaborador frente a sus compañeros de trabajo. Esto quiere decir que la norma exige la presentación de los resultados obtenidos en las evaluaciones de desempeño que se han realizado periódicamente, con las cuales se evidencie que el trabajador no ha cumplido con los indicadores y estándares mínimos de desempeño establecidos por la organización.

A este respecto, es importante mencionar que se deben construir y medir indicadores de desempeño productivo que influencien y determinen la posición estratégica y competitiva de la empresa en el mercado, de manera que estos tengan efecto sobre la motivación de los colaboradores a la hora de realizar las acciones de mejoramiento del desempeño. Los indicadores deben construirse de modo tal que eviten manipulaciones que consistan en mejorar el indicador en un área empeorando el desempeño en otras. También deben ser consistentes con las medidas de desempeño más amplias que utilice la organización. Por lo tanto, los indicadores de desempeño deben referirse, entre otros, a la calidad, la eficiencia, el ausentismo, el servicio al cliente, la seguridad y el desperdicio (Montuschi, 2019).

Por otro lado, en las empresas de servicios es muy difícil la medición adecuada de la productividad de los servicios por lo problemático que resulta definir el producto de una actividad de servicios y adicionalmente la noción de productividad. Esta noción se refiere a la relación entre la cantidad de productos obtenidos por un sistema productivo y los recursos utilizados para obtener aquella producción. Cuando esta se aplica a los servicios intangibles, enfrenta muchas dificultades por las características de los servicios, entre las cuales se tiene principalmente que se trata de un intangible y su evaluación es claramente subjetiva porque depende de la 
percepción del cliente. De ahí que, al aplicar las definiciones de productividad a la medición de servicios inmateriales, limitan su medición a parámetros cuantificables, tales como la cantidad de clientes atendidos en cierto período de tiempo, que terminan configurando incentivos perversos sobre la calidad del servicio y el reconocimiento al servicio realizado por el trabajador (Correal Espinal y Gutierrez Roa, 2017).

Por tanto, en relación con "los servicios, es fundamental el desarrollo de vínculos perdurables con los clientes, lo que implica que estén satisfechos, comprometidos con la relación y dispuestos a recomendar el servicio, lo cual se convierte en una ventaja competitiva" (Baptista y Leon, 2013, p. 194). De ahí que sean estos los elementos a los cuales se deben orientar los indicadores de desempeño en las empresas de servicios.

La finalidad de los cuadros comparativos que exige la norma en su literal b es dar total claridad al trabajador de que es la persona que está por debajo de la medida mínima establecida por la empresa para considerar como satisfactorio su nivel de desempeño y, adicionalmente, que está registrando los peores resultados de todo el equipo de trabajo a pesar de los plazos y recomendaciones que se le han dado para que mejore su rendimiento. De esta manera, se puede garantizar la objetividad e igualdad en las decisiones que sobre su desempeño se adoptan.

\section{Explicaciones y descargos del trabajador}

En el literal c del artículo 2 del Decreto 1373 (1966) se impone la obligación de citar a descargos al trabajador, es decir, se obliga a la apertura de un proceso disciplinario. De esta manera, se agrega una limitación adicional y exclusiva para esta causal de terminación del contrato, la cual obliga a dar cumplimiento a lo establecido por la Corte Constitucional en la Sentencia C 593 (2014), y cumplir con los siguientes requisitos mínimos para garantizar el derecho fundamental al debido proceso:

I. La comunicación formal de la apertura del proceso disciplinario a la persona a quien se imputan las conductas posibles de sanción.

II. La formulación de los cargos imputados, que puede ser verbal o escrita, siempre y cuando en ella consten de manera clara y precisa las conductas, las faltas disciplinarias a que esas conductas dan lugar y la calificación provisional de las conductas como faltas disciplinarias.

III. El traslado al imputado de todas y cada una de las pruebas que fundamentan los cargos formulados.

IV. La indicación de un término durante el cual el acusado pueda formular sus descargos, controvertir las pruebas en su contra y allegar las que considere necesarias para sustentar sus descargos.

V. El pronunciamiento definitivo del patrono mediante un acto motivado y congruente.

VI. La imposición de una sanción proporcional a los hechos que la motivaron. 
VII. La posibilidad que el trabajador pueda controvertir, mediante los recursos pertinentes, todas y cada una de las decisiones ya sea ante el superior jerárquico de aquél que impone la sanción como la posibilidad de acudir a la jurisdicción laboral ordinaria.

\section{Procedimiento para despedir por bajo desempeño}

En síntesis, se observa que el procedimiento que debe ejecutarse para dar cabal cumplimiento a lo establecido en el artículo 2 del Decreto 1373 (1966) y respetar los derechos fundamentales al debido proceso y al trabajo en condiciones dignas y justas, debe desarrollarse conforme a las siguientes etapas (figura 1):

- Al finalizar el periodo de evaluación de desempeño establecido por la empresa, se debe retroalimentar a las personas que constituyan el grupo conformado por el $5 \%$ de los empleados que obtienen los peores resultados. De esta manera, se configura la retroalimentación número 1.

- Si se detectan situaciones personales que afecten el desempeño de un trabajador en particular, se deben ajustar las condiciones de trabajo o de evaluación del mismo. De igual modo, se debe garantizar que el trabajador cuente con los elementos y condiciones de trabajo óptimas para cumplir a cabalidad con las funciones propias de su cargo.

- Finalizado el periodo siguiente de evaluación, se analizan los resultados de las personas retroalimentadas en el periodo inmediatamente anterior. Si sus resultados se mantienen y continúan en el grupo conformado por el $5 \%$ de los empleados que obtienen los peores resultados, se procederá a la realización de la retroalimentación número 2.

- Al realizar estas dos retroalimentaciones, se está dando cabal cumplimiento al requisito exigido por el literal a del artículo 2 del Decreto 1373 (1966).

- En estas circunstancias, si en el periodo de evaluación inmediatamente siguiente el trabajador continúa registrando un bajo desempeño que lo mantiene en el grupo equivalente al $5 \%$ de los empleados que obtienen los peores resultados, es procedente el inicio o apertura de un proceso disciplinario con base en los resultados obtenidos en este tercer mes de evaluación.

- Si la decisión a adoptar por el empleador es el despido, se debe verificar si el trabajador está inmerso en una condición de estabilidad laboral reforzada, en la cual la decisión quedará supeditada a la obtención de la autorización judicial o administrativa correspondiente.

Este procedimiento genera un efecto preventivo general en los demás trabajadores al observar las consecuencias de registrar bajo desempeño. Se crea, entonces, como lo explica Gómez Horta (2016), una conciencia jurídica general en virtud de la sanción 
a la que se exponen al reprobar las evaluaciones de desempeño. Con este ciclo procedimental se logra que los equipos progresen, toda vez que se mejora el desempeño. Por el contrario, si se mantienen resultados deficientes, se configura claramente una causal de terminación del contrato de trabajo (Bock, 2015).

De este modo, en cada ocasión que este procedimiento logre que un trabajador apruebe la evaluación de desempeño, se estará logrando mejorar la productividad de la empresa. Paralelamente, se estará preconstituyendo la prueba para demostrar la configuración de la justa causa de terminación del contrato conforme a las exigencias legales y jurisprudenciales ya analizadas en los casos donde el trabajador no logre mejorar sus indicadores de desempeño.
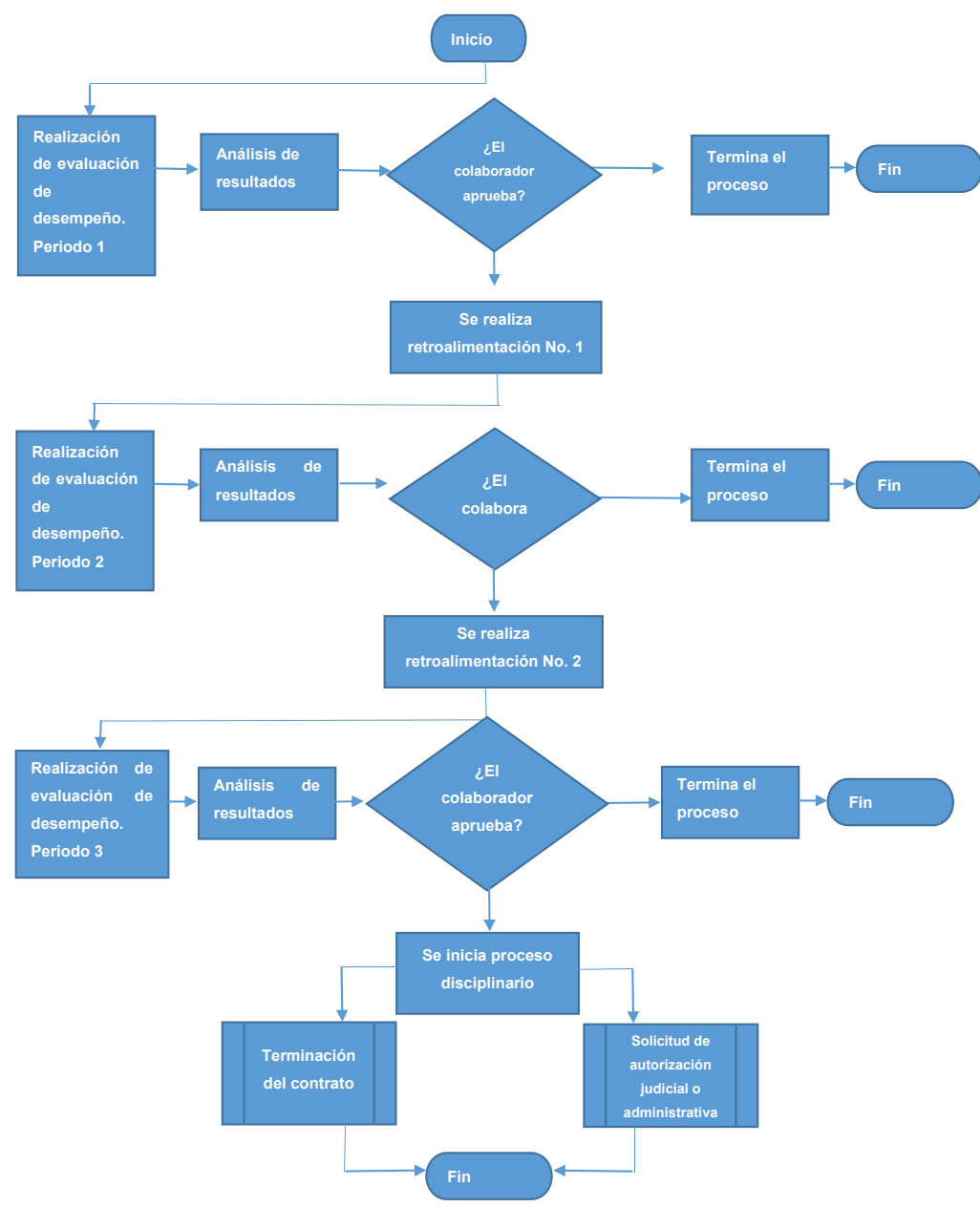

Figura 1. Flujograma procedimiento sancionatorio del bajo desempeño laboral Fuente: elaboración propia. 
Por último, en el presente estudio se considera necesario establecer algunas adiciones a los reglamentos internos de trabajo para dotar de mayor eficacia y seguridad estas decisiones. En este sentido, es importante que se establezca como justa causa de terminación del contrato una prohibición que podría ser en los términos que propongo a continuación:

Incumplir los objetivos, las metas y/o métricas de operación y/o gestión propuestas por el empleador y conocidas por el trabajador, durante tres periodos consecutivos y/o tres periodos en un semestre, aunque sean discontinuos. Los períodos de medición serán definidos libremente por el empleador de acuerdo con sus necesidades operativas y podrán ser iguales, mayores o menores a los periodos de medición anteriores, sin ninguna limitación.

Esta salvedad se haría con el propósito de prescindir de la obligación de preavisar la terminación del contrato establecida en el artículo 62 para los numerales 9 al 15 (Decreto 1373, 1966). De este modo, la causal legal que se aplica no es la contenida en el numeral 9, sino la contenida en el numeral 6 del citado artículo. Ahora bien, esto podría interpretarse como la forma de evadir el procedimiento exigido por el Decreto 1373 (1966). Sin embargo, esto no es adecuado si se atienden los fundamentos del proceso de evaluación de desempeño expuestos en el primer capítulo del presente artículo, toda vez que se pierde la posibilidad de evaluar el rendimiento de los trabajadores con relación a los niveles de productividad que requiere la empresa.

\section{CONCLUSIONES}

En primer lugar, respecto del análisis de la evaluación de desempeño se concluye que es una herramienta esencial para determinar el bajo rendimiento de los trabajadores para efectos de establecer si este bajo rendimiento conlleva la terminación del contrato de trabajo con justa causa. Esta circunstancia se debe probar con total objetividad en la medición y en la comparación con los resultados de los trabajadores entre sí y, por consiguiente, es la condición objetiva que se requiere para sancionar válidamente el bajo rendimiento laboral y garantizar los derechos fundamentales del trabajador al debido proceso y al trabajo en condiciones dignas y justas.

En segundo lugar, después del análisis de las limitaciones legales y jurisprudenciales desarrolladas respecto de la facultad del empleador de dar por terminado el contrato de trabajo unilateralmente, se concluye que para despedir válidamente un trabajador en virtud del bajo rendimiento laboral se debe indagar que el bajo rendimiento del trabajador no esté asociado a circunstancias personales, familiares, económicas y de salud que afectan las capacidades y la concentración del trabajador para lograr un óptimo desempeño. Luego de verificar que estas circunstancias personales, familiares, económicas y de salud que pueden afectar al trabajador no configuran una protección laboral reforzada que torne ineficaz el despido, se deben 
implementar los requerimientos dirigidos a informar el trabajador su bajo rendimiento y el inicio del plazo razonable para mejorar su desempeño. Finalmente, se debe desarrollar una investigación disciplinaria que garantice el derecho fundamental al debido proceso si el trabajador no logró mejorar su desempeño dentro del plazo razonable que le fue informado.

Finalmente, y con base en el procedimiento expuesto en el capítulo tercero del presente artículo, se concluye que será válido, seguro y eficaz terminar el contrato con justa causa si se da aplicación a este procedimiento, que tiene como insumo principal la evaluación del desempeño ejecutada en la empresa a partir de indicadores que miden la productividad de cada trabajador de forma objetiva. Esta evaluación, seguida por un proceso de retroalimentación, brinda herramientas para el mejoramiento del desempeño e indaga por aspectos propios de la esfera personal del trabajador que potencialmente afectan la prestación del servicio. De este modo, se garantiza su derecho fundamental al trabajo en condiciones dignas y justas.

Todo este proceso finaliza con un proceso disciplinario respetuoso del derecho fundamental al debido proceso que parte de todas las pruebas que demuestran al trabajador que no ha logrado cumplir los estándares mínimos de productividad exigidos por la empresa. Y que a pesar de que se le informó la situación y se le brindaron herramientas para que mejore su rendimiento, las evaluaciones de desempeño evidenciaron que no lo logró cumplir las expectativas mínimas, lo que configuraría una causal de terminación del contrato.

Por último, la decisión de despedir al trabajador después de desarrollar estas etapas concluye con una decisión válida que minimiza los riesgos laborales, toda vez que parte de una evaluación objetiva, respeta sus derechos fundamentales y aplica la normatividad que reglamenta esta casual de terminación del contrato.

\section{REFERENCIAS}

Arango, M. D., Ruiz, S., Ortiz, L. F. y Zapata, J. A. (2017). Indicadores de desempeño para empresas del sector logístico: Un enfoque desde el transporte de carga terrestre. Ingeniare. Revista Chilena de Ingeniería, 25(4), 707-720. https://dx.doi.org/10.4067/S0718-33052017000400707.

Baptista, M. V. y Leon, M. F. (2013). Estrategias de lealtad de clientes en la banca universal. Estudios Gerenciales, 29(127), 189 - 203. https://doi.org/10.1016/j.estger.2013.05.007

Bermudez, D., Martínez, M., Porte, D. y Safranov, A. (2016, 12 de julio). Características del Feedback Negativo [tesis de grado, Fundación UADES]. Repositorio Fundación UADE. https://repositorio.uade.edu. ar/xmlui/bitstream/handle/123456789/6056/Bermudez.pdf?sequence=4EisAllowed=y

Bock, L. (2015). La nueva fórmula del trabajo. Penguin Ramdom House.

Bohlander, G., Snell, S. y Herrero, D. M. (2008). Administración de Recursos Humanos. Cengage Learning.

Buitrago, L. (2013, 6 de junio). Contratación de servicios personales ¿decisión estratégica del estado colombiano? [ensayo para especialización, Universidad Militar Nueva Granada]. Repositorio Universidad 
Militar Nueva Granada. https://repository.unimilitar.edu.co/bitstream/handle/10654/11 197/ BuitragoMarquezLeonardo2013.pdf?sequence $=1$

Castillo, J. (1993). Administración de personal. Un enfoque hacia la calidad. Ecoe Ediciones.

Cequea, M., Rodriguez, C. y Nuñez, M. (2011). La productividad desde una perspectiva humana. Intangible Capital, 7(2), 549-584. http://www.redalyc.org/articulo.oa?id=54921605013

Chiang, M. M. y San Martín, N. J. (2017). Análisis de la Satisfacción y el Desempeño Laboral en los Funcionarios de la Municipalidad de Talcahuano. Ciencia y Trabajo, 17(54), 159-165. https://dx.doi. org/10.4067/S0718-24492015000300001

Chiavenato, I. (2008). Gestión del Talento Humano. McGrawHill.

Córdoba, C. y Moreno, M. (2013). Instrumento para Evaluar Variables Psicológicas y Laborales en Conductores de Autobuses. Reflexiones, 92(2), 109-122. https://revistas.ucr.ac.cr/index.php/ reflexiones/article/view/8826

Correal Espinal, A. y Gutierrez Roa, F. (2017). Medición de la Productividad en Empresas de Servicios y su Impacto en la Salud Mental de los Trabajadores. Memorias de la Décima Sexta Conferencia Iberoamericana en Sistemas, Cibernética e Informática, 190-195.

Corte Constitucional (1993, 27 de Octubre). Sentencia T-483/93 (Jose Hernandez MP). https://www. corteconstitucional.gov.co/relatoria/1993/t-483-93.htm

Corte Constitucional (1998, 19 de Octubre) Sentencia T-584/98 (Hernando Vergara MP) https://www. corteconstitucional.gov.co/relatoria/1998/T-584-98.htm

Corte Constitucional (2000, 05 de Abril) Sentencia C-386/00 (Antonio Barrera MP) https://www. corteconstitucional.gov.co/relatoria/2000/C-386-00.htm.

Corte Constitucional (2001, 08 de Junio) Sentencia T-611/01, (Jaime Cordoba MP) https://www. corteconstitucional.gov.co/relatoria/2001/T-611-01.htm

Corte Constitucional (2001, 24 de Octubre) Sentencia C-1110/01, (Clara Vargas MP). https://www. corteconstitucional.gov.co/relatoria/2001/C-1110-01.htm

Corte Constitucional (2014, 10 de Septiembre) Sentencia T-682/14 (Jorge Palacio MP) https://www. corteconstitucional.gov.co/relatoria/2014/T-682-14.htm.

Corte Constitucional (2014, 20 de Agosto). Sentencia C 593/14, (Jorge Pretelt MP) https://www. corteconstitucional.gov.co/RELATORIA/2014/C-593-14.htm

Corte Constitucional (2019, 29 de Octubre) Sentencia T-509/00 (Alejandro Cantillo MP). https://www. corteconstitucional.gov.co/relatoria/2019/t-509-19.htm

Corte Suprema de Justicia (2009, 4 de Agosto) Sentencia 35516 (Camilo Tarquino MP).

Corte Suprema de Justicia (2011, 30 de Agosto) Sentencia 40283 (Carlos Molina MP).

Corte Suprema de Justicia (2018, 18 de Abril). Sentencia SL1146 (Dolly Caguasango MP).

Corte Suprema de Justicia (2018, 24 de Abril). Sentencia SL1444 (Santander Brito MP)

Corte Suprema de Justicia (2018, 24 de Enero) Sentencia SL023 (Jorge Burgos MP).

Corte Suprema de Justicia (2018, 9 de Agosto) Sentencia SL3424, (Clara Dueñas MP)

Cuesta, A. (2017). Gestión del Talento Humano y del Conocimiento. Ecoe Ediciones. 
Cuesta, A., Fleitas, S., García, V., Hernández, I., Anchundia, A. y Mateus, L. (2018). Evaluación del desempeño, compromiso y gestión de recursos humanos en la empresa. Ingeniería Industrial, 34(1), 24-35. http://scielo.sld.cu/pdf/rii/v39n1/rii04118.pdf

Echeverria, R. (2003). Ontología del Lenguaje. JC Sáez Editor.

Eisele, P. (2012). Improving Performance in Groups. Baltic Journal of Psychology, 13 (1), 45-57. http:// dspace.lu.lv/dspace/bitstream/handle/7/1368/Balt-Psych-Journal_2012.pdf?sequence $=1$ EisAllo wed $=\mathrm{y} \#$ page $=45$

Fonseca, R. E. (2015). Competitividad, La Clave del Éxito Empresarial. Ed. Alfa y Omega.

Galindo, M. y Rios, V. (2015). Productividad. Serie de Estudios Económicos, 1(1), 2-9. https://scholar.harvard. edu/files/vrios/files/201508_mexicoproductivity.pdf.

Gomez, A. L. (1991). El primado de la razón práctica. Centro Editorial Universidad del Valle.

Gomez Horta, R. (2016). La prevención general y especial en el sistema penal y penitenciario colombiano. Summa Iuris, 4(1), 154-169. https://doi.org/10.21501/23394536.2087

Hernandez, L. (1997). Poder de Dirección del Empleador. En N. Buen Lozano, y E. Morgado Valenzuela, Instituciones de derecho del trabajo y de la seguridad social (pp. 405 419). Universidad Nacional Autónoma de México Instituto de Investigaciones Jurídicas.

Jaimes, L. y Rojas, M. D. (2015). Una mirada a la productividad laboral para las pymes de confecciones. Iteckne, 12(2), 177-187. http://www.scielo.org.co/pdf/itec/v12n2/v12n2a09.pdf

Juzgado 17 Laboral del Circuito de Cali (2019, 10 de Agosto) Sentencia 174, Radicado 76001310501720180015900 .

Medina, J. E. (2010). Modelo integral de productividad, aspectos importantes para su implementación. Revista EAN, 1(69), 110-119.

Montuschi, L. (2019). Salarios, productividad y competitividad: las negociaciones de empresa. https://ucema. edu.ar/publicaciones/download/documentos/180.pdf

Presidencia de la Republica de Colombia. (1950, 5 de agosto). Decreto Ley 2663. Código Sustantivo de Trabajo. http://www.secretariasenado.gov.co/senado/basedoc/codigo_sustantivo_trabajo.html

Presidencia de la Republica de Colombia. (1966, 26 de mayo). Decreto 1373 de 1966. Por el cual se reglamentan los artículos $4^{\circ}, 7^{\circ}$ numerales 9,14 y 15; $9^{\circ}, 10,14$, numeral 2; 17, 20, 25, 26, 30 y 40 del Decreto extraordinario número 2351 de 1965. http://www.suin-juriscol.gov.co/viewDocument.asp?ruta=Decretos/1275429

Rios Giraldo, R. M. (2016). El talento humano en los sistemas de gestión. Icontec.

Sanchez, J. y Calderón, V. (2012). Diseño del proceso de evaluación del desempeño del personal y las principales tendencias que afectan su auditoría. Pensamiento y Gestión, 16 (75), 54-82.

Smith, A. (1996). La riqueza de las naciones. Alianza Editorial.

Tejada, J. G. (2016). Debido proceso y procedimiento disciplinario laboral. Revista Opinión Jurídica Universidad de Medellín, 15(30), 227-248. https://doi.org/10.22395/ojum.v15n30all

Yepes, C. R. (2017). Por otro camino. Penguin Random House Grupo Editorial. 